innovation landscape. This is why dedicated forums and spaces that can mobilize alliances of companies, policymakers, academics and citizens are needed to raise the bar for responsible innovation and co-develop new mechanisms for self-governance on the business side alongside new government regulation, including the ones mentioned above. International organizations such as the OECD or IEEE are uniquely positioned to speak to differences in national regulations and are already playing key roles in fostering the necessary dialogs ${ }^{3,6,27}$. Universities, too, can mobilize their educational and entrepreneurial ecosystems to heighten sensitivity to responsibility concerns and foster policy dialog when companies are in the startup stage. What is more, experimental 'living lab' and 'sandbox' approaches could be used to co-develop new regulations and foster public debate about novel technologies, not just to create pro-business innovation environments through lower regulatory standards, as is currently the case in many such settings ${ }^{30}$. Neurotech companies, with obvious social and ethical challenges on the horizon, have a chance to set an example for the entire tech industry.

\section{Sebastian M. Pfotenhauer (iD) 1 , Nina Frahm (D), David Winickoff² David Benrimoh ${ }^{3,4}$, Judy Illes (D) ${ }^{5}$ and Gary Marchant ${ }^{6}$}

${ }^{1}$ Munich Center for Technology in Society (MCTS) and TUM School of Management, Technical University of Munich, Munich, Germany. ${ }^{2}$ Working Party for Bio-, Nano- and Converging Technologies, Organisation For Economic Co-operation and Development (OECD), Paris, France. ${ }^{3}$ Department of Psychiatry, McGill University, Québec, Montréal, Canada. ${ }^{4}$ Aifred Health, Montreal, Quebec, Canada. ${ }^{5}$ Neuroethics Canada, Department of Medicine,
University of British Columbia, Vancouver, British Columbia, Canada. ${ }^{6}$ Sandra Day O'Connor College of Law, Global Institute for Sustainability and Innovation, Arizona State University, Phoenix, AZ, USA.

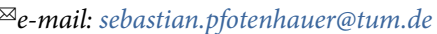

Published online: 7 June 2021

https://doi.org/10.1038/s41587-021-00947-y

\section{References}

1. Eaton, M. L. \& Illes, J. Nat. Biotechnol. 25, 393-397 (2007).

2. Jarchum, I. Nat. Biotechnol. 37, 993-996 (2019).

3. Garden, H., Winickoff, D., Frahm, N. \& Pfotenhauer, S. M. Responsible innovation in neurotechnology enterprises. OECD Science, Technology and Industry Working Papers, No. 2019/05 (OECD, 2019); https://doi.org/10.1787/9685e4fd-en

4. Wexler, A. A skeptic's take on Neuralink and other consumer neurotech. Stat (7 April 2021).

5. Wexler, A. \& Reiner, P. B. Science 363, 234-235 (2019).

6. OECD. OECD Recommendation on Responsible Innovation in Neurotechnology https://www.oecd.org/science/ recommendation-on-responsible-innovation-inneurotechnology.htm (2019).

Wexler, A. Front. Hum. Neurosci. 11, 224 (2017).

8. Schwartz, A. Science 350, 11 (2015).

9. Ienca, M., Haselager, P. \& Emanuel, E. J. Nat. Biotechnol. 36, $805-810$ (2018)

10. Ienca, M., Jotterand, F. \& Elger, B. S. Neuron 97, 269-274 (2018).

11. Nuffield Council on Bioethics. Novel Neurotechnologies: Intervening in the Brain. https://www.nuffieldbioethics.org/ publications/neurotechnology (2013).

12. Salles, A. et al. Neuron 101, 380-384 (2019).

13. Global Neuroethics Summit Delegates. et al. Neuron 100, 19-36 (2018)

14. De Stefano, V. Neuro-surveillance and the right to be human at work. On Labor https://onlabor.org/neuro-surveillance-andthe-right-to-be-humans-at-work/ (15 February 2020).

15. Clausen, J. et al. Science 356, 1338-1339 (2017).

16. Jones, O. D., Marois, R., Farah, M. J. \& Greely, H. T. J. Neurosci. 33, 17624-17630 (2013).

17. Asher-Schapiro, A. Out of my mind: advances in brain tech spur calls for 'neuro-rights'. Reuters (29 March 2021).

18. Ienca, M. \& Andorno, R. Life Sci. Soc. Policy 13, 5 (2017).

19. Marchant, G. \& Allenby, B. Bull. At. Sci. 73, 108-114 (2017).

20. Barben, D., Fisher, E., Selin, C. \& Guston, D. in The Handbook of Science and Technology Studies (eds Hackett, E. J. et al.) 979-1000 (MIT Press, 2008).

21. Stilgoe, J., Owen, R. \& Macnaghten, P. Res. Policy 42, $1568-1580$ (2013)

22. NIH. ELSI planning and evaluation history. https://www.genome. gov/10001754/elsi-planning-and-evaluation-history (2012).
23. Frahm, N. M. International governance of neuroscience and neurotechnology: whom to trust with the assessment of future pathways? The Neuroethics Blog http://www.theneuroethicsblog. com/2017/11/international-governance-of.html (2017).

24. Pfotenhauer, S.M., Laurent, B., Papageorgiou, K. \& Stilgoe, J. Soc. Stud. Sci. (in the press).

25. AUTM. In the Public Interest: Nine Points to Consider in Licensing University Technology https://www.autm.net/AUTMMain/media/ Advocacy/Documents/Points_to_Consider.pdf (2007).

26. Benrimoh, D., Israel, S., Perlman, K., Fratila, R. \& Krause, M. in Recent Trends and Future Technology in Applied Intelligence (eds Mouhoub, M. et al.) 869-880 (Springer International, 2018); https://doi.org/10.1007/978-3-319-92058-0_83

27. IEEE. Ethically Aligned Design, Version 1, Translations and Reports https://standards.ieee.org/industry-connections/ec/ead-v1.html (2016).

28. Kurtz, E. in The Oxford Handbook of Corporate Social Responsibility (eds Crane, A. et al.) 249-280 (Oxford Univ. Press, 2008); https://doi.org/10.1093/oxfordhb/9780199211593. 003.0002

29. Smith, N. M., Zhu, Q., Smith, J. M. \& Mitcham, C. Sci. Eng. Ethics 27, 28 (2021).

30. Engels, F., Wentland, A. \& Pfotenhauer, S. M. Res. Policy 48, 103826 (2019).

\section{Acknowledgements}

S.P. acknowledges support by the European Union's Horizon 2020 research and innovation program under grant agreement 788359 ('SCALINGS: Scaling up co-creation? Avenues and limits for including society in science and innovation'). J.I. is supported by the Canada Research Chairs Program as the Canada Research Chair in Neuroethics and co-leads the Canadian Brain Research Strategy (CIHR Grant \#171583;03027 IC-127354).

\section{Competing interests}

S.P. serves as academic program director for the master's program 'Responsibility in Science, Engineering, and Technology' (REST) at Technical University of Munich. D.W. is a senior policy analyst at the Organisation for Economic Co-operation and Development (OECD), where he heads the Working Party for Biotechnology, Nanotechnology and Converging Technologies (BNCT) responsible for the Recommendation on Responsible Innovation in Neurotechnology. D.B. is a founder, shareholder and employee of Aifred Health, a digital mental health company.

\section{Additional information}

Peer review information Nature Biotechnology thanks the anonymous reviewers for their contribution to the peer review of this work.

\title{
The need for global access to biomedical innovations during pandemics
}

To the Editor - The COVID-19 pandemic has fundamentally reshaped the way in which research and development is conducted and, in the case of vaccines and therapeutics, compressed a decade-long product development process to less than one year ${ }^{1}$. However, the new technologies produced by this accelerated innovationsuch as mRNA vaccines, viral vector vaccines, monoclonal antibodies and state-of-the-art diagnostics-have not been equitably distributed worldwide. Intense early competition among high-income and upper-middle-income countries over initially scarce supplies has prevented lowand middle-income countries (LMICs) from accessing these innovations ${ }^{2}$. For vaccines, this problem will likely continue if annual vaccinations are required and/or SARS-CoV-2 variants warrant modification and subsequent boosters-both requiring additional supplies. The lack of delivery systems to distribute these innovations in many LMICs further compounds this situation $^{3,4}$. At best, most populations in LMICs will face a protracted delay of months to years in receiving these innovations at scale; at worst, some populations may never receive them ${ }^{5,6}$.

To effectively respond to pathogens with pandemic potential, the product development ecosystem needs to both develop biomedical products in an even shorter timeframe and distribute these products globally in an equally rapid manner. Building on proposed strategies 
to further accelerate product development for global infectious diseases ${ }^{7}$, a concrete plan is needed to define the path for those products in LMICs-from initial regulatory approvals through to the last step of delivery to patients. Here, I argue that the key players in the global health ecosystem-funders, industry, international agencies, foundations, and national and supranational governments-must work together to provide resource-poor countries with purchasing power and implementation capacity that is on par with those of resource-rich countries.

\section{The failure of global access}

The national protectionist behavior exhibited by governments in wealthy countries during COVID-19 is nothing new. Resource-rich nations acted in just the same way in 2009 during the H1N1 (swine flu) pandemic ${ }^{8}$. The playbook runs as follows: large advanced purchase commitments (APCs) obtained from vaccine and therapeutics manufacturers secure most of the initial supply of product; rich countries acquire a staggeringly disproportionate amount of doses much earlier than LMICs. Without available competitive capital, LMICs cannot compete with wealthy countries in procuring critical biomedical innovations. International organizations then attempt to mobilize resources to assist LMICs and, at best, secure a fraction of the overall supply in a delayed timeframe.

With COVID-19, the COVID-19 Vaccines Global Access Facility (COVAX) was designed to recruit high-income and upper-middle-income countries as 'self-financing' participants that would (i) buy enough vaccine doses via the facility to initially vaccinate up to $50 \%$ of their own populations and (ii) provide financial support for an APC of doses for LMICs to vaccinate up to $20 \%$ of their populations. The up-front financing matched with pooled demand would dramatically increase COVAX's competitiveness to bid for early doses from companies.

In practice, the voluntary nature of COVAX resulted in two crippling obstacles for the initiative: wealthy countries bypassed COVAX, securing vaccine doses through their own direct APCs with vaccine manufacturers; at the same time, wealthy countries under-delivered on their financing promises for COVAX in the needed timeframe. As a result, COVAX was effectively pushed to the back of the line for acquiring doses of certain vaccines, with the first approved vaccines being snapped up by wealthy nations. And, unlike wealthy countries, COVAX was unable to initally procure a diverse portfolio of vaccines, thus risking a complete lack of vaccine access should safety, efficacy or supply issues arise (all three have emerged in varying degrees as obstacles). Some LMICs-seeing no light at the end of the tunnel-have begun negotiating APCs outside of COVAX. Others are limiting vaccine exports and, consequently, delaying the availability of the majority of COVAX's near-term supply.

The World Bank's Pandemic Emergency Financing Facility was another financial mechanism designed to assist resource-poor countries in the event of a pandemic. Created before COVID-19, it comprised catastrophic insurance coverage and bonds. Unfortunately, its meager payouts and onerous restrictions provided too little capital too late, and the facility was abandoned in the middle of the COVID-19 pandemic after distribution of the initial funds?

The Access to COVID-19 Tools Accelerator, another large but voluntary funding effort focusing on delivering new tools (vaccines, diagnostics and therapeutics) and strengthening health systems, has suffered a similar fate: it has a funding shortfall of at least $\$ 23$ billion to reach its goals of ensuring equitable access to new COVID-19 biomedical technologies.

While 778 million vaccines doses have been administered globally, only 14 million (1.8\%) have been administered in Africa. Equally sobering, monoclonal antibody use in LMICs has been virtually non-existent ${ }^{10}$.

\section{Global access via mandated transnational cooperation}

The world is at an unprecedented point in our ability to respond to pandemics. We can perform near-real-time emerging pathogen surveillance. We can rapidly develop biomedical tools to address identified pathogens. But we are missing a third critical component to effectively predict and prevent pandemics, which is ensuring global access to these tools. As the world becomes more interconnected, the human toll and the financial impact of emerging infectious diseases become more significant. The impact of the 2003 severe acute respiratory syndrome (SARS) epidemic, 2009 H1N1 swine flu pandemic, and 2014-2016 Ebola outbreak is estimated at \$40-55 billion each ${ }^{11}$. For 2021 alone, the economic impact of COVID-19 may reach $\$ 9.2$ trillion $^{12}$. Furthermore, letting viral disease run rampant in one region is a poor epidemiological strategy as this increases the probability of variants emerging and spreading, threatening the gains made with vaccination efforts in other countries. The earlier COVID-19 and future outbreaks and epidemics are contained globally, the more likely catastrophic economic damage can be averted.
The question is how to avoid a repeat of the practical failure of previous global access efforts in anticipation of the next major global health crisis. The key is shifting from an ad hoc, reactive approach to a deliberate, proactive strategy. This shift, I suggest, could occur through the following three actions.

\section{Financially empower low-income} countries. A pandemic procurement fund should be established through a combination of traditional voluntary approaches and non-traditional mandated approaches that ensures consistent, permanent financing. In the event of an outbreak, epidemic or pandemic, this fund can immediately act as a pooled procurement negotiating group on behalf of LMICs when resource-wealthy countries begin their own nationally circumscribed negotiations. Beyond immediate-needs procurement, this fund could finance a global stockpile of essential biomedical technologies (like Ebola vaccines) and early manufacturing activities to expand the needed global supply capacity. This approach would overcome previous limitations by facilitating faster initial product availability and thus earlier pathogen containment.

\section{Implement essential operational}

processes. Pandemic-appropriate regulatory, manufacturing and distribution mechanisms need to be defined and established for LMICs, as any delay in these steps will impede an efficient and effective response ${ }^{13}$. In an outbreak or pandemic, it is unclear for both new and existing platform technologies what regulatory mechanisms will be needed for each resource-poor country and what accelerated regulatory pathways may be available. Harmonizing regulatory requirements, including a standardized pharmacovigilance system, across many countries would minimize the burden faced by countries and developers and may be possible through initiatives like the African Medicines Agency. To expand the global supply capacity for biologics-based technologies, regional manufacturing hubs could capture either end-to-end manufacturing or specific aspects of production, including source materials and essential components. Ultimately, these could serve as the primary source of supply for resource-poor countries and overcome single-supplier constraints, but would need to be kept active through the production of non-pandemic products. In-country rapid distribution mechanisms should be pilot tested on a regular basis to ensure they execute effectively during the actual time of need ${ }^{14}$. As with product development efforts for 
COVID-19 that were conducted in parallel, these product implementation mechanisms also need to be developed in parallel as they are essential but need to be established as soon as possible.

Deliver multiple innovations over time. Widespread use of the first phase of technologies for an outbreak or pandemic may be constrained by implementation obstacles (for example, ultracold storage or intravenous administration), but it is critical that such initial innovations are delivered in resource-poor countries, even if only to a small proportion of the target population. This would allow not only the commencement of an effective in-country response, but also pilot testing of new technologies and the identification and fixing of implementation issues. As reflected in most infectious diseases, vaccines and therapeutics undergo multiple iterations to optimize properties, such as formulation, frequency of dosing, thermostability, and number of doses. Diagnostic technologies also undergo similar product iteration cycles. The rapid rise of new SARS-CoV-2 lineages may already require adaptation of some existing technologies to target a greater proportion of divergent variant strains or regularly emerging strains ${ }^{15}$; for example, the introduction of pan-coronavirus technologies similar to polyserotype Streptococcus pneumoniae vaccines or annually modified vaccines similar to those for influenza. Indeed, two vaccines that constitute a large proportion of the initial signed, binding supply agreements for COVAX appear to have reduced efficacy against the B.1.351 SARS-CoV-2 variant, originating in South Africa $^{16-18}$. Biomedical innovation access strategies for resource-poor countries should account for iterative product development and the need to implement a wide range of innovations over time.

Influenza gives us an annual reminder of the global nature of infectious diseases, and COVID-19 has confirmed how an outbreak in one country can morph into a pandemic within weeks. We can now likely detect an impending pandemic earlier than ever and develop the necessary biomedical tools faster than previously thought, but achieving true global health security necessitates an aggressive approach to deliver those innovations globally as quickly as possible. Building a global product development ecosystem that addresses access in resource-poor settings is a both a socially just and financially prudent strategy. It will help the world improve its response to the COVID-19 pandemic and be better prepared for the next pandemic.

\section{Rajesh Gupta (1D) \\ Vir Biotechnology, San Francisco, CA, USA.

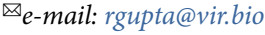

Published online: 24 May 2021

https://doi.org/10.1038/s41587-021-00942-3

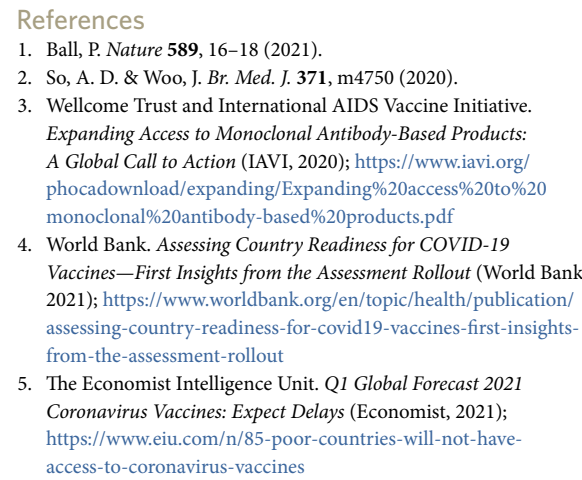

6. Feinberg, M. Enabling equitable and affordable access to monoclonal antibodies for COVID-19. Workshop on Allocation of COVID-19 Monoclonal Antibody Therapies and Other Novel Therapeutics (National Academies Press, 2020); https://www.nationalacademies.org/event/12-16-2020/docs/ DC6D3EEAB5BDDEA427A1B1368564CE08502058A833CC

7. Gupta, R. Science 370, 913-914 (2020)

8. Fidler, D. P. PLoS Med. 7, e1000247 (2010)

9. Bump, J. B., Friberg, P. \& Harper, D. R. Br. Med. J. https://doi. org/10.1136/bmj.n180 (2021).

10. Sanders, K. et al. Monoclonal antibodies for COVID-19 in Africa: a complex web of opportunities and challenges. Health Affairs Blog (2 March 2021); https://www.healthaffairs.org/do/10.1377/ hblog20210224.158341/full/

11. Global Preparedness Monitoring Board. A World At Risk (World Health Organization, 2019); https://apps.who.int/gpmb/assets/ annual report/GPMB annualreport_2019.pdf

12. Cakmakli, C. et al. The Economic Case for Global Vaccinations (International Chamber of Commerce Research Foundation, 2021); https://iccwbo.org/publication/ the-economic-case-for-global-vaccinations/

13. Simpson, S. et al. Lancet Infect Dis. https://doi.org/10.1016/S1473 3099(20)30123-7 (2020).

14. Johns Hopkins Center for Health Security. Clade X Exercise: Improving Policy to Prepare for Severe Pandemics (Johns Hopkins Center for Health Security, 2018); https://www. centerforhealthsecurity.org/our-work/events/2018_clade_x exercise/pdfs/Clade-X-executive-summary-document.pdf

15. Burton, D. R. \& Topol, E. J. Nature 590, 386-388 (2021).

16. Madhi, S. A. et al. N. Eng. J. Med. https://doi.org/10.1056/ NEJMoa2102214 (2021).

17. Shinde, V., et al. N. Eng. J. Med. https://doi.org/10.1056/ NEJMoa2103055 (2021).

18. Gavi-the Vaccine Alliance. COVAX Global Supply Forecast (GAVI, 2021, accessed 10 April 2021); https://www.gavi.org/sites/ default/files/covid/covax/COVAX\%20Supply\%20Forecast.pdf

\section{Acknowledgements}

The author thanks David Heymann, Peter Hotez, Shonali Shome, Charles Wells and Gavin Yamey for insights. This article was prepared by the author acting in a personal capacity. The views and opinions expressed in this article are those of the author and do not necessarily reflect the official policy or position of Vir Biotechnology, nor that of any other individual, employee, agency, organization, or company associated with Vir Biotechnology.

\section{Competing interests}

R.G. is an employee of and may own shares in Vir Biotechnology, Inc., which is developing vaccines and therapies for global infectious diseases.

\section{Tracking cell lineages to improve research reproducibility}

To the Editor - Human cell lines are central to biomedical research and medicine, but genetic evolution and inconsistencies among derived lineages are too often ignored. These issues are becoming increasingly important now that wide adoption of gene editing technologies such as CRISPR has led to a boom in the development of new genetic lineages with knock-in reporters or patient-specific mutations (Fig. 1a). A more detailed view of cell line provenance and lineage formation can guard against wasted research effort and funds and, ultimately, improve reproducibility of biological research. Accurate cell line tracking is also required for safely establishing cell therapies for precision medicine.

Currently, $18-36 \%$ of common cell lines are estimated to be mislabeled or contaminated; in addition, cell lines often evolve divergent lineages ${ }^{1,2}$. Cell lineages can form by spontaneous or induced selection events during cell culture or when cells are genetically modified. Although funders and journals are starting to acknowledge the importance of cell line authentication, cell lineage provenance is rarely recorded or published, despite its impact on data reliability and reproducibility ${ }^{3-5}$.

Here, we discuss lineage divergence as a natural, inevitable phenomenon across all kingdoms of life. We highlight how lineage formation in the culture of human cells is influenced by routine laboratory practices and has accelerated in the genomics and gene-editing era. We also propose simple changes to working routines to minimize unwanted lineage divergence. Lastly, we explore how monitoring divergence 